\title{
Cavity linewidth narrowing with dark-state polaritons
}

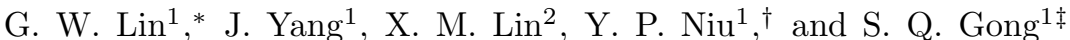 \\ ${ }^{1}$ Department of Physics, East China University of Science and Technology, Shanghai 200237, China and \\ ${ }^{2}$ School of Physics and Optoelectronics Technology, \\ Fujian Normal University, Fuzhou 350007, China
}

\begin{abstract}
We perform a quantum-theoretical treatment of cavity linewidth narrowing with intracavity electromagnetically induced transparency (EIT). By means of intracavity EIT, the photons in the cavity are in the form of cavity polaritons: bright-state polariton and dark-state polariton. Strong coupling of the bright-state polariton to the excited state induces an effect known as vacuum Rabi splitting, whereas the dark-state polariton decoupled from the excited state induce a narrow cavity transmission window. Our analysis would provide a quantum theory of linewidth narrowing with a quantum field pulse at the single-photon level.
\end{abstract}

\section{INTRODUCTION}

Electromagnetically induced transparency (EIT) can be used to make a resonant, opaque medium transparent by means of a strong coupling field acting on the linked transition [1, 2]. The EIT medium in an optical cavity known as intracavity EIT was first discussed by Lukin et al. [3]. They show that the cavity response is drastically modified by intracavity EIT, resulting in frequency pulling and a substantial narrowing of spectral features. By following this seminal work, significant experimental advance has been made in narrowing the cavity linewidth [4-7] and enhancing the cavity lifetime [8].

The previous semi-classical treatments of cavity linewidth narrowing and lifetime enhancing with intracavity EIT were based on the solution of the susceptibility of EIT system. Here we present a quantumtheoretical treatment of cavity linewidth narrowing with intracavity EIT. By means of intracavity EIT, the photons in the cavity are in the form of cavity polaritons: bright-state polariton and dark-state polariton. Strong coupling of the bright-state polariton to the excited state induces an effect known as vacuum Rabi splitting, whereas the dark-state polariton decoupled from the excited state induces a narrow cavity transmission window $v=\cos ^{2} \theta v_{0}$, with $v_{0}$ the empty-cavity linewidth and $\theta$ the mixing angle of the dark-state polariton [9]. We discuss the condition required for cavity linewidth narrowing and find that when the atom-cavity system is in the collective strong coupling regime, a weak control field is sufficient for avoiding the absorption owing to spontaneous emission of the excited state, and then the darkstate polariton induces a very narrow cavity linewidth. This result is different from that based on previous semiclassical treatments of intracavity EIT [3, 4], where the strong control field is required for avoiding the absorption of a probe field pulse if all atoms are prepared in one

\footnotetext{
*Electronic address: gwlin@ecust.edu.cn

${ }^{\dagger}$ Electronic address: niuyp@ecust.edu.cn

‡Electronic address: sqgong@ecust.edu.cn
}
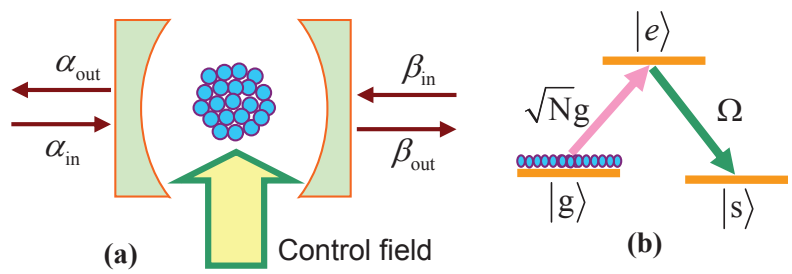

(b)

FIG. 1: (Color online) (a) Schematic setup to cavity linewidth narrowing with dark-state polaritons. (b) The relevant atomic level structure and transitions.

of their ground states.

\section{SEMI-CLASSICAL THEORY}

We first review the intracavity EIT with the semiclassical theory based on the solution of the susceptibility of EIT system. An EIT medium of length $l$ is trapped in an optical cavity of length $L$. The EIT medium response of a probe classical field is characterized by the real $\chi^{\prime}$ and the imaginary $\chi^{\prime \prime}$ parts of the susceptibility. The real part gives the dispersion $\frac{\partial \chi^{\prime}}{\partial \omega_{p}}$ and the imaginary part gives the absorption coefficient $\alpha=2 \pi \omega_{p} \chi^{\prime \prime} / c$, with probe frequency $\omega_{p}$. Then the ratio of the linewidth $v$ of the cavity with the EIT medium to that of the empty cavity is $[3,4]$

$$
\frac{v}{v_{0}}=\frac{1-r \tau}{\sqrt{\tau}(1-r)} \frac{1}{1+\eta}
$$

where $\tau=\exp (-\alpha l), \eta=\omega_{r}(l / 2 L) \frac{\partial \chi^{\prime}}{\partial \omega_{p}}$, with $\omega_{r}$ the cavity resonant frequency, and $r$ is the intensity reflectivity of the cavity mirror. As shown in Ref [3, 4], when the EIT medium is driven by a strong control field, the absorption of the probe field can be negligible $\left(\chi^{\prime \prime} \rightarrow 0\right)$, whereas the dispersion is large, resulting in a substantial narrowing of the cavity linewidth. 


\section{QUANTUM-THEORETICAL TREATMENT}

Now we try to solve the quantum dynamics of EIT in an optical standing-wave cavity [Fig, 1(a)]. We consider an atomic system with three levels, two ground states $|g\rangle,|s\rangle$, and an excited $|e\rangle$, forming a $\Lambda$-configuration [see Fig, 1(b)]. The two transitions $|g\rangle \leftrightarrow|e\rangle$ and $|s\rangle \leftrightarrow|e\rangle$ are resonantly coupled by a cavity mode and a laser fields, respectively. The interaction Hamiltonian for the coherent processes is described by $H_{I}=$ $\sum_{j=1}^{N}\left(g|e\rangle_{j}\langle g|a+\Omega| s\rangle_{j}\langle e|+H . c.\right)$, where $a$ is the annihilation operator of the cavity mode, $g(\Omega)$ is the coupling strength of quantized cavity mode (external field) to the corresponding transition. We assume that almost all atoms are in one of their ground states, e.g. $|G\rangle=\prod_{j=1}^{N}\left|g_{j}\right\rangle$, at all times, and define the collective atomic operators $C_{\mu}^{\dagger}=\frac{1}{\sqrt{N}} \sum_{j=1}^{N}|\mu\rangle_{j}\langle g|$ with $\mu=e, s$, then $H_{I}$ can be rewritten as

$$
H_{I}^{\prime}=\sqrt{N} g C_{e}^{\dagger} a+C_{s}^{\dagger} C_{e} \Omega+H . c .
$$

where the coupling constant $g$ between the atoms and the quantized cavity mode is collectively enhanced by a factor $\sqrt{N}$. In analogy to the dark-state polariton for a travelling light pulse [9], one can define two standing-wave cavity polaritons: a dark-state polariton $m_{D}=\cos \theta a-\sin \theta C_{s}$, and a bright-state polariton $m_{B}=\sin \theta a+\cos \theta C_{s}$, with $\cos \theta=\Omega_{c} / \sqrt{N g^{2}+\Omega_{c}^{2}}$ and $\sin \theta=\sqrt{N} g / \sqrt{N g^{2}+\Omega_{c}^{2}}$. In terms of these cavity polaritons the Hamiltonian $H_{I}^{\prime}$ can be represented by

$$
H_{I}^{\prime \prime}=\sqrt{N g^{2}+\Omega^{2}}\left(C_{e}^{\dagger} m_{B}+C_{e} m_{B}^{\dagger}\right)
$$

The cavity dark-state polariton is decoupled from the collective excited state $C_{e}^{\dagger}|G\rangle$.

The external fields interact with cavity mode $a$ through two input ports $\alpha_{i n}, \beta_{i n}$, and two output ports $\alpha_{\text {out }}, \beta_{\text {out }}$. The Hamiltonian for the cavity input-output processes is described by [10] $H_{\text {in-out }}=\sum_{\Theta=\alpha, \beta} \int_{-\infty}^{+\infty} \omega d \omega \Theta^{\dagger}(\omega) \Theta(\omega)+$ $\sum_{\Theta=\alpha, \beta}\left[i \int_{-\infty}^{+\infty} d \omega \sqrt{\frac{\kappa}{2 \pi}} \Theta^{\dagger}(\omega) a+H . c.\right]$, where $\omega$ is the frequency of the external field, $\kappa$ is the bare cavity decay rate without EIT medium, and $\Theta(\omega)$ with the standard relation $\left[\Theta(\omega), \Theta^{\dagger}\left(\omega^{\prime}\right)\right]=\delta\left(\omega-\omega^{\prime}\right)$ denotes the one-dimensional free-space mode. We express the Hamiltonian $H_{\text {in-out }}$ in the polariton bases:

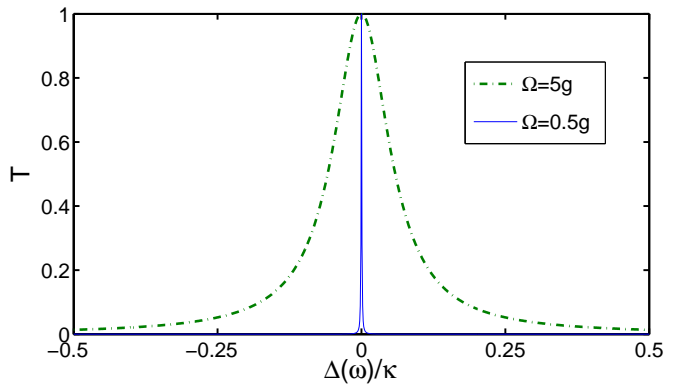

FIG. 2: (Color online) The transmission spectrum $T$ as a function of $\Delta(\omega)$ for $\Omega=\{5 g, 0.5 g\}$, on the assumption that $N=400, g=\kappa=\gamma_{e}$.

$$
\begin{aligned}
H_{\text {in-out }}^{\prime} & =\sum_{\Theta=\alpha, \beta} \int_{-\infty}^{+\infty} \omega d \omega \Theta^{\dagger}(\omega) \Theta(\omega) \\
& +\sum_{\Theta=\alpha, \beta}\left[i \int _ { - \infty } ^ { + \infty } d \omega \Theta ^ { \dagger } ( \omega ) \left(\sqrt{\frac{\kappa_{D}}{2 \pi}} m_{D}\right.\right. \\
& \left.\left.+\sqrt{\frac{\kappa_{B}}{2 \pi}} m_{B}\right)+H . c .\right],
\end{aligned}
$$

with $\kappa_{D}=\cos ^{2} \theta \kappa$ and $\kappa_{B}=\sin ^{2} \theta \kappa$.

In the intracavity EIT system, only the bright-state polariton $m_{B}$ resonantly couples to the excited state. Under the condition

$$
\sqrt{N g^{2}+\Omega^{2}} \gg \kappa_{B}, \gamma_{e},
$$

with $\gamma_{e}$ the spontaneous-emission rate of the excited state $|e\rangle$, the resonant interaction in Hamiltonian $H_{I}^{\prime \prime}$ in the socalled strong coupling regime will induce an effect known as vacuum Rabi splitting [11], i.e., the splitting of the cavity transmission peak for the bright-state polariton $m_{B}$ into a pair of resolvable peaks at $\omega=\omega_{0} \pm \sqrt{N g^{2}+\Omega^{2}}$ (here $\omega_{0}$ is the resonant frequency of cavity mode). Thus one can neglect bright-state polariton $m_{B}$ to calculate the cavity transmission spectrum near the cavity resonant frequency $\omega_{0}$.

According to quantum Langevin equation, the evolution equation of the cavity dark-state polariton $m_{D}$ is given by [10]

$$
\dot{m}_{D}=-i \omega_{0} m_{D}-\kappa_{D} m_{D}+\sqrt{\kappa_{D}} \alpha_{i n}+\sqrt{\kappa_{D}} \beta_{i n} .
$$

Using the relationships between the input and output modes at each mirror [10]

$$
\alpha_{\text {out }}(t)+\alpha_{\text {in }}(t)=\sqrt{\kappa_{D}} m_{D},
$$

and

$$
\beta_{\text {out }}(t)+\beta_{\text {in }}(t)=\sqrt{\kappa_{D}} m_{D},
$$




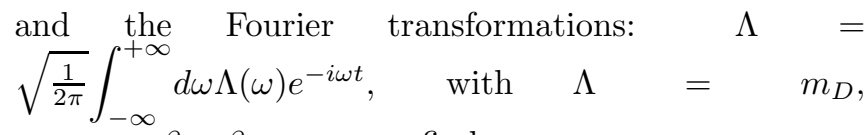
$\alpha_{\text {in }}, \alpha_{\text {out }}, \beta_{\text {in }}, \beta_{\text {out }}$, we can find

$$
\alpha_{\text {out }}(\omega)=\frac{\kappa_{D} \beta_{\text {in }}(\omega)}{\kappa_{D}-i \Delta(\omega)}
$$

where $\Delta(\omega)=\omega-\omega_{0}$, and we have assumed that the photons enter into the cavity from the input port $\beta_{\text {in }}$ $\left(\alpha_{i n}=0\right)$. Then the transmission spectrum for intracavity EIT is described by

$$
T(\omega)=\frac{\left|\alpha_{\text {out }}(\omega)\right|^{2}}{\left|\beta_{\text {in }}(\omega)\right|^{2}}=\frac{\kappa_{D}^{2}}{\kappa_{D}^{2}+\Delta^{2}(\omega)} .
$$

As depicted in Fig. 2, the transmission spectrum $T$ can be controlled by the external coherent field. Then the calculate of cavity linewidth $\Delta_{v}$, i.e., the full width at half height of $T(\omega)$, gives

$$
v=2 \kappa_{D}=2 \kappa \cos ^{2} \theta=\cos ^{2} \theta v_{0},
$$

here $v_{0}=2 \kappa$ is the empty-cavity linewidth [10].

\section{BRIEF DISCUSSION}

Next we briefly discuss the results of the quantumtheoretical treatment of intracavity EIT. First, the polariton $m_{D}$ corresponds to the well-known "dark-state polariton" for a travelling light pulse [9]. In Ref [9], the mixing angle $\theta$ determines the group velocity of darkstate polariton, whereas the mixing angle $\theta$ of cavity dark-state polariton $m_{D}$ here determines the effective cavity decay rate $\kappa_{D}$. Second, the atomic ground states have long coherence time and very small decay rate, thus the main source of absorption by EIT system is spontaneous emission of the excited state. To avoid the absorption of the probe field by the coupling of bright-state polariton $m_{B}$ to excited state, we require the condition in Eq. (5). If atom-cavity system is in the weak coupling regime $\sqrt{N} g<\kappa, \gamma_{e}$, we need a strong control field,
$\Omega \gg g$, to satisfy the required condition. However, if atom-cavity system is in the collective strong coupling regime $\sqrt{N} g \gg \kappa, \gamma_{e}$, even when the control field is so weak that $\Omega \ll g$, the required condition is still satisfied and the absorption owing to spontaneous emission of the excited state can be neglected, then the dark-state polariton will induce a very narrow cavity linewidth $v=$ $\cos ^{2} \theta v_{0} \approx v_{0} \Omega^{2} / N g^{2}$. We note this result is different from that based on previous semi-classical treatments of intracavity EIT [3, 4], where a strong control field is required for avoiding the absorption of a probe field pulse if initially (i.e., before the probe field arrives) all atoms are in their ground states $|G\rangle=\prod_{j=1}^{N}\left|g_{j}\right\rangle$.

\section{CONCLUSION}

In summary, we have performed a theoretical investigation of intracavity EIT quantum mechanically. In intracavity EIT system the cavity photons are in the form of cavity polaritons: bright-state polariton and dark-state polariton. Strong coupling of the bright-state polariton to the excited state leads to an effect known as vacuum Rabi splitting, whereas the dark-state polariton decoupled from the excited state induce a narrow transmission window. If atom-cavity system is in the weak coupling regime, a strong control field is requied for avoiding the absorption owing to spontaneous emission of the excited state. However, if atom-cavity system is in the collective strong coupling regime, a weak control field is sufficient for avoiding the absorption, and then the darkstate polariton induces a very narrow cavity linewidth. This result is different from that based on previous semiclassical treatments of intracavity EIT [3, 4], where the strong control field is required for avoiding the absorption of a probe field pulse if all atoms are prepared in one of their ground states. Our quantum-theoretical treatment of intracavity EIT would provide a quantum theory of linewidth narrowing with quantum field pulses at the single-photon level.

Acknowledgments: This work was supported by the National Natural Sciences Foundation of China (Grants No. 11204080, No. 11274112, No. 91321101, and No. 61275215), the Fundamental Research Funds for the Central Universities (Grants No. WM1313003).
[1] S. E. Harris, "Electromagnetically induced transparency," Phys. Today 50, 36 (1997).

[2] M. Fleischhauer, A. Imamoglu, and J. P. Marangos, "Electromagnetically induced transparency: optics in coherent media," Rev. Mod. Phys. 77, 633 (2005).

[3] M. D. Lukin, M. Fleishhauer, M. O. Scully, and V. L. Velichansky, "Intracavity electromagnetically induced transparency," Opt. Lett. 23, 295 (1998).

[4] H. Wang, D. J. Goorskey, W. H. Burkett, and M. Xiao, "Cavity-linewidth narrowing by means of electromagnetically induced transparency," Opt. Lett. 25, 1732 (2000).
[5] H. B. Wu and M. Xiao, "Cavity linewidth narrowing and broadening due to competing linear and nonlinear dispersions," Opt. Lett. 32, 3122 (2007).

[6] G. Hernandez, J. P. Zhang, and Y. F. Zhu, "Vacuum Rabi splitting and intracavity dark state in a cavity-atom system," Phys. Rev. A 76, 053814 (2007).

[7] H. B. Wu, J. Gea-Banacloche, and M. Xiao, "Observation of Intracavity Electromagnetically Induced Transparency and Polariton Resonances in a DopplerBroadened Medium," Phys. Rev. Lett. 100, 173602 (2008). 
[8] T. Lauprêtre C. Proux, R. Ghosh, S. Schwartz, F. Goldfarb, and F. Bretenaker, "Photon lifetime in a cavity containing a slow-light medium," Opt. Lett. 36, 1551 (2011).

[9] M. Fleischhauer and M. D. Lukin, "Dark-State Polaritons in Electromagnetically Induced Transparency," Phys. Rev. Lett. 84, 5094 (2000).

[10] D. F. Walls, and G. J. Milburn, Quantum Optics
(Springer-Verlag, Berlin, 1994).

[11] A. Boca, R. Miller, K.M. Birnbaum, A. D. Boozer, J. McKeever, and H. J. Kimble, "Observation of the Vacuum Rabi Spectrum for One Trapped Atom," Phys. Rev. Lett. 93, 233603 (2004). 